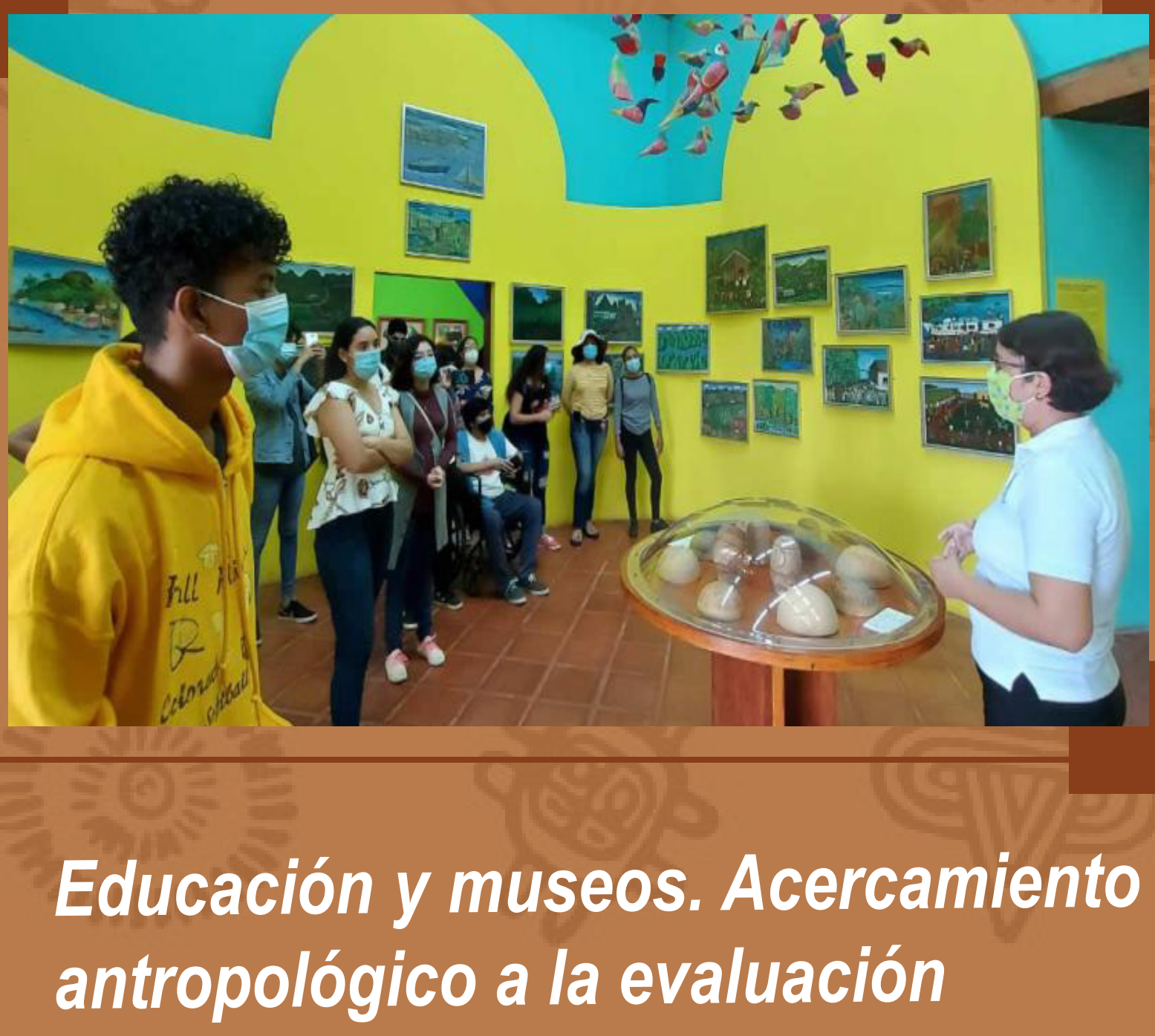

Antropología: Experiencias Educativas 


\title{
Educación y museos. Acercamiento antropológico a la evaluación
}

\section{Education and museums. Anthropological approach to evaluation}

\author{
José Luis Ramos $\mathrm{R}$
}

Escuela Nacional de Antropología e Historia, México

ID Orcid: https://orcid.org/0000-0002-2261-5095

xozeluizr@gmail.com

\section{Resumen}

En el presente artículo expongo algunos puntos críticos acerca de la evaluación en museos, desde la antropología, enfocados a la educación no-formal. Un primer aspecto refiere al falso dilema planteado en los estudios de público de una oposición entre investigar y evaluar, considerando que la segunda corresponde a un tipo de investigación aplicada, que cumple con los requisitos y condiciones básicas de cualquier pesquisa. Investigación que tiene el carácter de ser comparativa, aspecto que no siempre es enunciado con claridad. Enseguida señalo la importancia del contexto, poco atendido, en el campo educativo, cultural y museológico. Más adelante, recomiendo utilizar definiciones claras y explícitas sobre los aspectos a evaluar, de lo contrario se generan resultados confusos o dudosos. Un siguiente punto - ligado al tema central de la XVII Camarilla - corresponde a la difícil tarea de evaluar la educación no-formal que ocurre en los museos. Ítems que en conjunto van a expresar la importancia de la perspectiva antropológica, en términos conceptuales y metodológicos. Por último, cierro el texto con una reflexión final.

Palabras claves: Evaluación, educación no-formal, museos, investigación aplicada y comparativa

\section{Abstract}

In this article I expose some critical points about museum evaluation, from anthropology, focused on non-formal education. A first aspect refers to the false dilemma raised in public studies of an opposition between research and evaluation, considering that the second corresponds to a type of applied research, which meets the basic requirements and conditions of any research. Research that has the character of being comparative, an aspect that is not always clearly stated. Immediately I point out the importance of the context, little attended, in the educational, cultural and museum fields. Later, I recommend using clear and explicit definitions of the aspects to be evaluated, otherwise confusing or doubtful results are generated. A next point - linked to the central theme of the XVII Camarilla corresponds to the difficult task of evaluating non-formal education that occurs in museums. Items that together will express the importance of the anthropological perspective, in conceptual and methodological terms. Finally, I close the text with a final reflection.

Keywords: Evaluation, non-formal education, museums, applied and comparative research

1 Profesor-investigador, Titular C, adscrito a la Licenciatura en Etnología. ORCID: 0000-0002-2261-5095.

Encuentro entre los encargados del área educativa de diversos museos del país, que tuvo lugar en la Ciudad de Tlaxcala, Tlax., en octubre de 2017.

La ponencia expuesta sirvió de base para elaborar el presente artículo. 


\section{Introducción}

En los estudios de público o museológicos es constante la invitación a realizar pesquisas más integrales, frente a investigaciones parciales y paralelas que guardan distancia u oposición entre ellas (Rosas, 2017 y Zuazúa, 2017). Un ejemplo es el "dilema" entre investigar y evaluar, entendidas como dos formas opuestas de trabajo. Otros estudiosos promueven una labor que unifique las dos opciones; sin embargo, esto implica seguir pensando que son dos polos (Pérez, 2000). Por lo que prefiero considerar a la evaluación como un tipo de investigación aplicada y comparativa, que cumple los requisitos y condiciones básicas de indagar, manteniendo su particularidad.

Un aspecto importante para lograr una adecuada evaluación es el contexto. Diversos estudios lo dejan de lado centrando su labor en el plano metodológico o sólo técnico (el diseño y aplicación de los instrumentos), sin reconocer que el contexto determina el sentido de una evaluación. Además, está la falta de claridad en la definición de los conceptos para delinear los objetos de evaluación, provocando dudas sobre los resultados generados.

Un cuarto ítem apunta a la constante referencia sobre la educación escolar (formal) (Sánchez, 2015 y Viladot, 2009), minimizando la de carácter no-formal. A partir de ella se establecen criterios y rubros por evaluar, cuando la educación no-formal es sustancialmente distinta a la escolar y más compleja (Alderoqui y Pedersoli, 2011). A lo largo del texto voy a desarrollar con más detalle estos tres aspectos marcados.

\section{Falso dilema}

En el campo de la investigación científica existen diferentes criterios para establecer tipologías, ubicando particularidades y distinciones que existen en los procesos, niveles o formas de indagar, es un asunto heurístico; pero no significa que el investigador deba elegir una frente a otra en cuanto opciones cerradas, como lo plantean algunos interesados en los estudios de público al señalar el dilema entre investigar y evaluar (Pérez, 2000). No alude a una disputa epistemológica entre formas alternativas de conocer, ni de identificar lo que históricamente aparece como más pertinente. La "oposición" radica en el grado de exigencia metodológica que presenta cada una, ubicando a la evaluación en menor rango.

Criterio de distinción insuficiente para hablar de un dilema, por ello considero que la evaluación corresponde a un tipo de investigación aplicada, donde los objetivos para su empleo marcan la diferencia. También produce conocimiento, aunque sea local; situación semejante a otras modalidades de investigación (etnografía, teoría fundamentada o estudios de caso). La evaluación pretende incidir en la realidad estudiada, conforme a dos premisas: a) mejorarla y b) a través de ofrecer información (resultados de la pesquisa) que permita a las personas tomar decisiones adecuadas de cambio (Schmilchuk, 2017).

Definición que me invita a entenderla desde la antropología, particularmente la aplicada. Recordando a Balandier, que consideraba como la aculturación planificada "[...] que va a convertirse en el objetivo de la antropología aplicada, asume formas diversas [...] 
según el contexto en que haya de desenvolverse $[\ldots]^{\prime \prime}(1977,57)$. Me permite delinear a la evaluación como un tipo de investigación aplicada comparativa, cuyo objeto de estudio es el cambio sociocultural dirigido. Su tarea es generar conocimiento local sobre el cambio sociocultural provocado de manera intencional dentro de un contexto político nacional, que para el caso de los museos hablo de políticas, programas y proyectos culturales.

\section{Evaluación: investigación comparativa}

Hay dos aspectos a destacar: uno, el objeto de conocimiento es el cambio y, dos, su estudio consiste en comparar un antes y un después, incluyendo lo que ocurrió entre los dos momentos para comprender la diferencia. En tanto conocimiento local una opción metodológica es la etnografía.

Alternativa que presenta modalidades y niveles que pautan la minuciosidad o amplitud que requiera el evaluador, siempre referido a la unidad social (el museo). Enfoque que obliga a conocer el punto de vista de los sujetos involucrados en la estructura, organización y actividades dentro del museo (Ramos, 2017a). Condición que empata con la perspectiva que abrió la nueva museología.

El contraste me permite elaborar un esquema en donde lo sustancial es comparar dos situaciones a las que llamaré S1 y S2, además de marcar la acción dirigida (AD) que existe entre los dos momentos. Así, la tarea investigativa corresponde a una pesquisa comparativa (evaluativa): S1 - AD - S2. Donde la primera labor consiste en describir la situación inicial (S1), en un tiempo-1; diagnosticar (evaluación inicial) las condiciones y características que expresan la problemática a ser modificada. Después, indicar la serie de actividades programadas y operadas (recursos empleados, etc.) para lograr el cambio deseado (AD). Por último, anotar las nuevas condiciones y características de la situación final (S2), en un tiempo-2, y compararlas con las de la situación 1, que permita reconocer la modificación ocurrida entre ellas, señalando el grado alcanzado en el cambio planeado y dirigido.

Para identificar de manera clara la transformación experimentada es relevante el diagnóstico o evaluación inicial. Para reconocer la eficiencia del programa cultural convendrá indicar puntualmente las actividades llevadas a cabo, contrastadas con las originalmente planeadas en el diseño. De esta forma, la acción comparativa -estrategia central de evaluación- posibilita apreciar el nivel de cambio logrado, como la diferencia entre lo planeado y su operación.

\section{Contexto de evaluación}

A varios lectores les parecerá que no hay nada especial en el contenido del bosquejo anterior, que es de uso frecuente por diversos evaluadores; sin embargo, al revisar informes de evaluación y artículos publicados, es notorio advertir la ausencia de una labor comparativa adecuada, incluso que no hubo tal actividad. Volveré a este asunto más adelante, ahora me interesa atender otra carencia que considero fundamental en una evaluación -que le da sentido-, me refiero al contexto en que es planeada.

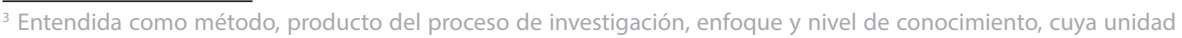
social de estudio será el museo (Ramos, 2017b). 


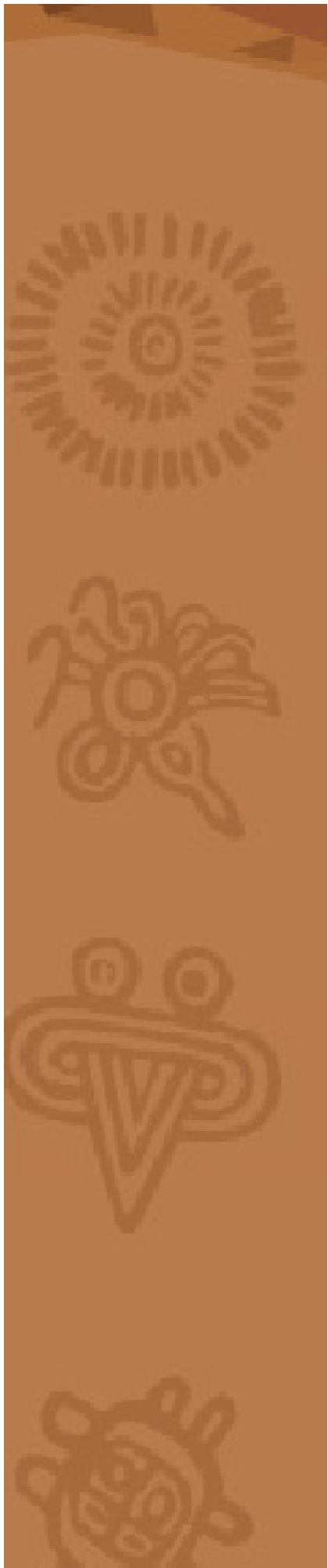

Contexto que responde a cuatro interrogantes: a) quién considera necesario llevar a cabo una evaluación, b) qué se pretende obtener, c) ligada a la anterior, qué uso tendrán los resultados de la evaluación y d) quién participará en esta labor, de manera directa e indirecta.

En un principio anoté varios considerandos en torno a la pesquisa evaluativa. Es un tipo de investigación aplicada con el propósito de mejorar o atender una problemática, advertida en la realidad social, empleando el conocimiento científico. Ofrece información de los resultados alcanzados conforme al plan de acción operado, permitiendo identificar necesidades que avalen las decisiones de mejoría en el programa de que se trate.

Para un adecuado diseño de evaluación es menester saber (contextualizar) quién es el sujeto específico (individual o colectivo) interesado en evaluar el programa o proyecto. Es un representante de la política pública en cuestión o son los operarios del programa o los directamente afectados, ¿quién requiere de un trabajo de evaluación? La influencia que ejerce en la tarea evaluativa corresponde a las preocupaciones propias de cada actor social mencionado, de sus intereses particulares, de lo que buscan encontrar (segunda interrogante contextual). Y, sobre todo, de sus pretensiones respecto a los resultados de la evaluación. Recordemos que se busca evaluar un cambio sociocultural intencional y dirigido, que modifica una determinada situación a través de una acción planificada.

Una tercera interrogante es quién va a diseñar y operar el proyecto de evaluación. Será cubierto por un agente externo o por los propios sujetos involucrados en la situación, a manera de autoevaluación. Sin olvidar que la unidad social no es una entidad homogénea, es factible que esté conformada por individuos que ocupan diferentes estatus, generando perspectivas diferentes al interior de la misma organización sociocultural.

Atender estas cuestiones va a permitir visualizar con mayor claridad el perfil específico que tendrá el proyecto de evaluación. Que en el caso de la actividad de y en los museos, importa considerar que forman parte de una política cultural nacional, donde es necesario tomar determinadas decisiones, enmarcadas en una dinámica compleja de intereses y negociaciones entre distintos actores sociales y políticos. Si bien, los procesos actuales de globalización y de conflictos internos nacionales obligan a repensar los estados nacionales, aún son una referencia importante en las políticas culturales que dan sentido a los proyectos museológicos en el país.

\section{De lo implícito a lo explícito}

Habiendo señalado la importancia del contexto de evaluación en el proyecto mismo, regreso al nivel metodológico de diseño.

Otra limitante fundamental en la evaluación es que no siempre son definidos claramente los conceptos que amparan el objeto a evaluar; son empleados de manera implícita, impidiendo una mayor puntualidad tanto en el proceso como en los resultados. Por ello, aunque parezca evidente o lógico, insisto en la necesidad de precisar el contenido conceptual de los términos empleados, explicitando los modelos ideales que subyacen en varios proyectos. Condición que me permite modificar el primer esquema a uno más amplio: MI / S1 - AD S2. 
La premisa apunta a reconocer la existencia de un modelo ideal (MI) de situación, pero que no siempre es anunciado; por el contrario, existe un silencio al respecto. En cambio, hacerlo visible posibilita identificar varias combinaciones razonables donde opera la tarea metodológica y sistemática de comparar.

El modelo ideal sostiene el discurso que emiten los evaluadores al establecer la situación como debería de ser, pero que en un inicio está ausente o limitada, lo que justifica la necesidad de una acción que permita acceder a la situación ideal. Intervención que debe ser planeada y operada conforme a la idea primigenia. Modelo ideal que aparece como una imagen ideológica (ii) que caracteriza un faltante, pero sin ampararse en evidencias. Puede ser presentada por cualquiera de los tres actores: directivos, operadores y beneficiarios. De esta forma, la comparación de resultados (en la situación 2) será con respecto a la imagen ideológica (ii) de una determinada problemática: ii / SI - AD - S2.

En otra versión, donde sí opera un "diagnóstico", se ofrece y justifica con evidencias una decisión previamente acordada; es decir, las evaluaciones iniciales son a modo para legitimar una decisión tomada. La comparación será entre los resultados obtenidos y el “diagnóstico" aplicado, por lo que la evaluación contendrá una importante carga ideológica.

Ahora bien, cuando existe un genuino interés en evaluar las metas alcanzadas en un programa, la comparación es entre los resultados logrados y la situación inicial diagnosticada, donde el modelo ideal permea implícitamente el discurso que anuncia el diagnóstico. No existen definiciones claras para reconocer el sentido de los términos empleados en el diagnóstico. Lo recomendable es que el modelo ideal sea explicitado con las definiciones conceptuales puntuales. Esto permite comparar la situación inicial y final, además contrastar con el modelo ideal que sustenta a la evaluación. Cada fase evaluativa será comparada ante el modelo ideal.

Con ello se establecen de manera clara y puntual los procedimientos evaluativos, fincando la base para el diálogo necesario entre todos los actores que participan en la experiencia museológica.

\section{Educación}

Siguiendo con el enfoque de reconocer los modelos ideales a evaluar, es menester acotar lo correspondiente a la educación no-formal. Para lo cual es necesario señalar algunos puntos críticos de la función educativa de y en los museos.

Para entender a la educación no-formal (no escolar), la estrategia recurrente ha sido compararla con la educación formal (escolar), estableciendo sus diferencias; sin embargo, veladamente existe un tránsito implícito hacia una distinción jerárquica, donde la educación escolar queda por encima de la educación no-formal. Por ejemplo, al marcar presencias y ausencias en la educación no-formal es en base al parámetro de la educación escolar, nunca se procede a la inversa, que la educación no-escolar sirva de base para la comparación. 
Un punto crítico estriba en la reducción que sufre el concepto e imagen de educación, siendo muy notoria al momento de la evaluación educativa. A pesar de emplear el término de educación, los especialistas están pensando en la educación escolar (profesores, pedagogos y psicólogos educativos) lo que es evidente al momento de querer evaluar. Situación que se exporta a los espacios no-escolares, como los museos. Otra forma reductiva aparece en la llamada evaluación educativa, al momento de reconocer logros, avances, problemas, etc., se transita, sin ninguna aclaración, a evaluar el aprendizaje. Lo mismo ocurre con la planeación de las estrategias didácticas, están pensadas en términos de aprendizaje escolar (Rubiales, 2011).

Algunos estudiosos reconocen que evaluar el aprendizaje sólo en términos cognitivos (aún más reducido) es limitado, por lo que proponen incluir aspectos sociales y emotivos. Pretenden generar un recorrido inverso, de lo específico transitar hacia algo más integral, pero continúan sin evaluar lo educativo (Sánchez, 2015). El contenido del concepto de educación ha sido excluido de la evaluación. Podemos entender su discurso académico traduciendo por aprendizaje la palabra educación y no pierde sentido su argumentación.

Otra manera de minimizar lo educativo en los museos se refleja en la limitada atención otorgada en los estudios de visitantes. Por ejemplo, en los estudios de público promovidos al interior del INAH (Instituto Nacional de Antropología e Historia) por cerca de dos décadas, son pocas las variables educativas incluidas en sus formatos de encuesta. De un total de 60 variables únicamente 9 remiten a posibles contenidos educativos. Y, la más específica (conocimiento adquirido) únicamente está presente en 29 (48.3\%) de los 75 estudios realizados entre 1998 y 2016 (INAH, 2016).

Un tercer punto crítico por mencionar, previo al esbozo de una posible evaluación de lo educativo no-formal, corresponde a la función de discriminación y exclusión que alienta la educación escolar, siendo su principal instrumento la evaluación. A partir ésta surgen los reprobados parciales y totales, rezagados, desertores, etc. Un fenómeno emparentado corresponde a la distinción entre instituciones escolares, las de prestigio con estudiantes que alcanzan notas altas y las estigmatizadas que cuentan con un perfil evaluativo completamente opuesto, provocando que aspirantes (y padres de familia) decidan esforzarse por ingresar a una escuela del primer tipo y eludan o experimenten como castigo formar parte de las de segundo orden.

Al revisar estas inflexiones, conviene generar una labor evaluativa específica para la educación "no-formal" (o informal), que atienda adecuadamente su carácter complejo, dinámico y flexible, intentando no ser excluyente y tomando en cuenta el contexto pautado por determinadas políticas culturales y educativas.

\section{Delineando un enfoque antropológico}

El contenido de los apartados previos, expresan la pertinencia e importancia de brindar una perspectiva antropológica para impulsar procesos de evaluación de la actividad educativa no-formal promovida en y desde los museos. Relevancia en términos teóricos, conceptuales y metodológicos. 
Un primer paso, consiste en ofrecer un concepto antropológico de educación diferente al empleado por pedagogos y psicólogos educativos. Propongo entenderla como un proceso sociohistórico de transmisión, adquisición y apropiación cultural (Ramos, 2009). Donde la cultura la remito como un sistema de ideas, normas, valores y afectos.

Proceso que involucra mínimamente a dos sujetos, un educador (transmisor) y un educando (adquiriente) y el contenido cultural variará conforme al momento histórico en que ocurra. Es decir, el conjunto de ideas, normas, valores y afectos, así como la estructura y organización en que ocurren los procesos educativos, contienen una variación temporal y social. Cada situación educativa cambiará conforme a la combinación particular de los ejes espaciales y temporales.

Su complejidad responderá al contexto sociohistórico en que ocurra, conforme al grado estructural y organizacional que muestre. La unidad social de estudio puede incluir diversos estatus sociales con sus respectivas lógicas culturales que están en una interacción dinámica, en constante negociación. Lo que implica analizar cada experiencia educativa sin necesidad de emplear el modelo binario de educación formal y no-formal, ambas situaciones son educativas.

Actividad educativa que es pensada, organizada y aplicada en el contexto de ciertas políticas culturales, se trata de una práctica educativa intencionada; es decir, de un cambio cultural dirigido, asunto que ha sido un rubro de interés para la investigación antropológica, en su vertiente aplicada. De tal manera que la mirada puede atender las ideas, prescripciones y valores tanto del staff como de los usuarios de un museo.

Un eje metodológico constante en la investigación antropológica es la comparación, pautada por la frecuente combinación entre lo universal-particular, para identificar lo común y la diferencia. Consideración, que me permitió advertir la carencia en las labores evaluativas en museos de no explicitar cuál es la imagen ideal implícita sobre la que pretenden diagnosticar (primera etapa de un proceso de evaluación) la situación en que se encuentran.

Con esta serie de elementos es que puedo hablar de ir delineado un enfoque antropológico de la evaluación, como una investigación aplicada comparativa sobre el cambio cultural dirigido que ocurre en los museos.

\section{Reflexión final}

Para evaluar (investigar comparativamente) una situación educativa "dentro" de un museo (físico o virtual) deben tenerse presentes tres cuestiones: aclarar el contexto de la evaluación, explicitar el modelo educativo ideal que ampara el quehacer de los evaluadores y cuidar que exista una correspondencia adecuada entre el marco conceptual y el desarrollo metodológico-técnico de la evaluación. 


\section{Referencias Bibliográficas}

Alderoqui, S. y C. Pedersoli (2011). La educación en los museos. De los objetos a los visitantes, Argentina: Paidós.

Balandier, R. (1977). Antropología aplicada, Argentina: Amorrortu.

Garza, Y. (2012). "La evaluación: su impacto en las prácticas” en: La evaluación educativa: análisis de sus prácticas, México: UNAM/CONACYT/Díaz de Santos.

González, R. (2012). "Aspectos clave de las perspectivas teóricas para la evaluación de programas educativos: México" en: La evaluación educativa: análisis de sus prácticas, México: UNAM/CONACYT/Díaz de Santos.

Pérez, E. (2000). Estudios de visitantes en museos. Metodología y aplicaciones, España: Trea.

Ramos, J.L: (2009). “Antropología, Cultura y Educación. Veredas interdisciplinarias” en: Matices. Revista de Posgrado, Año 4, No. 9, UNAM/FES-Aragón, México.

Ramos, J.L. (2017a). "Estudios etnográficos y etnológicos de público" en: Gaceta de Museos, Tercera Época, No. 67, Abril-julio, INAH, México.

Ramos, J.L (2017b). "Etnografía concéntrica y didáctica. Notas para no-antropólogos" en: Revista Nóesis, No. 52, UACJ, (México).

Rosas, A. (2017). "Públicos: historia y contemporaneidad" en: Estudios sobre públicos y museos. Vol. II. Apuntes para pasar de la teoría a la práctica. ENCRYM/Publicaciones digitales, México.

Rubiales, R. (2012). Modelo Educativo. Ciudad Juárez, Museo de la Revolución en la Frontera.

Sánchez, C. (2015). "La dimensión educativa de los museos de ciencias" en: Aportaciones a la museología y la educación en México, México: UNAM. 
Schmilchuk, G. (2017). "Dos caras de una moneda. Evaluar exposiciones y museos/ conocer públicos" en: Estudios sobre públicos y museos. Vol. II. Apuntes para pasar de la teoría a la práctica, ENCRYM/Publicaciones digitales, México.

Viladot, P. (2009). “¿Para qué vienen? Expectativas de los docentes en las visitas escolares al museo" en: Enseñanza de las ciencias, Revista de investigación y experiencias didácticas, Número Extra, VIII Congreso Internacional sobre Investigación en Didáctica de las Ciencias, Barcelona.

Zuazúa., B. (2017). “Análisis cronológico de la evolución de los estudios de públicos en México y el mundo. Una visión introductoria" en: "Estudios de públicos. Definición, áreas de aplicación y escalas" en: Estudios sobre públicos y museos. Vol. I. Públicos y museos: ¿Qué hemos aprendido? ENCRYM/Publicaciones digitales, México.

José Luis Ramos $\mathrm{R}$

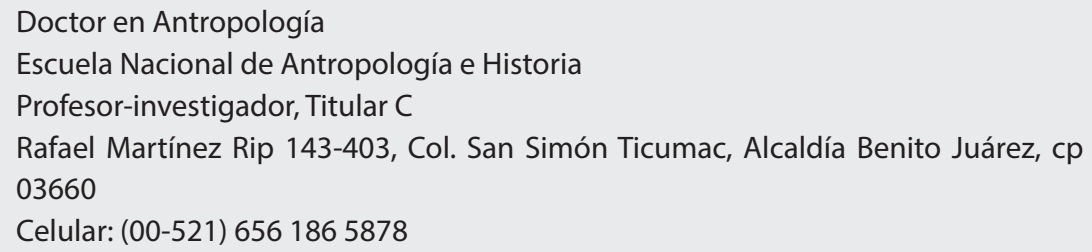

